\title{
Comunicación en salud
}

\section{Communication in health}

\author{
Lina Paola Bonilla-Mahecha1(i) \\ ${ }^{1}$ Servicio de Medicina Física y Rehabilitación, Hospital Militar Central, \\ Bogotá, Colombia
}

Urol Colomb 2020;29:174-175.

Dear Editor,

La actual situación de salud, a razón de la Declaración de la Pandemia del SARS - COV2, nos plantea la necesidad de reconocer y reflexionar sobre los procesos de comunicación de las instituciones y de los profesionales de la salud en tiempos de incertidumbre. Hemos sido testigos en los últimos meses del posicionamiento del tema en la agenda mediática, del aumento exponencial de los contenidos, así como de diferentes ciclos y procesos comunicacionales asociados al COVID - 19.

Sin embargo, poco se conoce en el contexto de la salud de los aspectos teóricos y metodológicos del enfoque de la comunicación de la gestión del riesgo o de la comunicación del riesgo. Proceso que consistente en la respuesta efectiva de las emergencias de salud pública, que depende de la capacidad de gestión, de la voluntad, de la disposición y de la regularidad para asumir los temas contemporáneos en salud. ${ }^{1-3}$

Por supuesto, las acciones y las estrategias en comunicación en salud, dependen del tema y de diferentes aspectos como el lenguaje, la forma, los canales, entre otros, que determinan la configuración de las intervenciones o contenidos en salud, por ejemplo, el esquema de vacunación de niños y niñas, en comparación, a la prevención e identificación temprana del cáncer de próstata.

En este punto, me permito dirigirle una pregunta y extenderla a sus lectores, ¿somos conscientes de la importancia e impacto que se puede generar a partir de las acciones en comunicación en un contexto de riesgo en salud, en este caso, de la Pandemia SARS - COV2?

Permítame contextualizar a sus lectores...

Primero que todo, no existe una definición única de la comunicación del riesgo, sin embargo, me acojo a los abordajes interdisciplinarios que pretenden orientar los esfuerzos en salud bajo dos principios básicos, credibilidad y veracidad de la información. De acuerdo con la Organización Mundial de la Salud (OMS) la comunicación del riesgo, emplea diferentes técnicas y estrategias de comunicación, involucra las percepciones y conocimientos de las pobla-

received

June 25, 2020

accepted

July 7, 2020
DOI https://doi.org/

$10.1055 / \mathrm{s}-0040-1715520$.

ISSN 0120-789X.

e ISSN 2027-0119.
Address for correspondence Lina Paola Bonilla-Mahecha, Magíster, Hospital Militar Central, Tv. 3, 49-02, Bogotá 111161375, Colombia (e-mail: Ipbonillam@unal.edu.co).

ciones, y, se refiere al intercambio de información, participación de expertos temáticos en la emisión de opiniones en tiempo real frente a situaciones que amenazan la salud, la economía y el desempeño social de las comunidades. ${ }^{4}$

En adición, los tomadores de decisiones y los profesionales de la salud, deben contar y proveer la especificidad de los contenidos técnicos (propios del tema y/o en asociación a la especialidad clínica que se re requiera), así como emitir normas, guías, protocolos y rutas de atención de acuerdo a la mejor evidencia disponible; generar acciones informativas eficaces, caracterizadas por mensajes claros, con baja posibilidad de inferencia o interpretaciones alternativas; y divulgar por canales de comunicación temporales y/o flexibles de acuerdo a las necesidades de información.

Lo anterior, requiere comprender la atemporalidad de los enunciados, es decir, divulgar en tiempo real las recomendaciones, además de contar con un equipo de comunicación con experiencia en el sector de la salud, a partir de lo cual, se expandan y aprovechen las virtudes del enfoque de la comunicación del riesgo, en tanto, este cumple unos ciclos y tiempos específicos de corta duración que deben prever la articulación con otros enfoques y modelos como la Educación en salud, Edu-entretenimiento, Precede - Procede, Comunicación para el Cambio Social y el Comportamiento (CCSYC), entre otros, con el fin de continuar con los objetivos de comunicación en salud de las instituciones y de los profesionales.

Sin duda alguna, conocer y adherirse al enfoque de la comunicación del riesgo, debe ser un proceso ético y responsable, en el que invito a que no se asuma como una acción de "divulgación de información vertical", sino que se convierta en una oportunidad para reconocer las percepciones, valores, comportamientos y prácticas en salud de las personas, comprender la diversidad de los canales de información en los que consultan, y, dinamizar nuestras acciones de comunicación, sin detrimento de la calidad y de la oportunidad de acceso a la información.

Todo lo anterior, permitirá, aportar en la toma de decisiones informadas para mitigar los efectos de la amenaza o el

Copyright (C) 2020, Sociedad Colombiana License terms de Urología. Publicado por Thieme Revinter Publicações Ltda., Rio de Janeiro, Brazil. Todos los derechos reservados. 
riesgo, implantar e implementar medidas y acciones de protección y prevención, reducir la propagación del riesgo, salvar vidas, ${ }^{5}$ así como, educar - comunicar en salud desde una visión amplia, flexible y acorde a las necesidades y contextos que nos exige el siglo XXI.

La comunicación del riesgo, requiere de un abordaje interdisciplinario, en tanto, la gestión, las amenazas, el grado de exposición y las vulnerabilidades de cada estructura cultural, determinan los estilos y concepciones del riesgo, los cuales deben conducir las acciones de gestión y prevención adecuadas, ${ }^{6}$ en las que prime la necesidades de comunicar - educar, de manera clara y oportuna, además de reconocer la diversidad en términos de interlocución, accesibilidad, disponibilidad y acceso tecnológico, creatividad e innovación, entre otros, alejándonos de una lectura reduccionista, centrada en la forma y no en el contenido.

Finalmente, la situación actual es una oportunidad para orientar y aportar, en la toma de decisiones en salud, desde el acercamiento a los indicadores, el impacto y la efectividad, en dialogo con la Comunicación - Salud - Cultura, ${ }^{7}$ como una apuesta metodológica para el reconocimiento y validación del otro, la interacción y la dinamización multilenguaje y multimedia, que configuran la experiencia en comunicación y educación en salud basada en evidencia; a partir de la cual reflexionemos sobre nuestro rol en la configuración de las nuevas ciudadanías en salud y los aprendizajes aprendidos a partir del actual contexto que el vivimos a causa del COVID - 19.
Conflicto de Intereses

Los autores declaran no tener ningún conflicto de intereses.

\section{Referencias}

1 World Health Organization. Best practices for communicating with the public during an outbreak - Report of the WHO. 2005: 21-23http://www.who.int/iris/handle/10665/69138

2 Lundgren R, Mcmak A. Risk Communication: a handbook for communicating, environmental, safety, and health risk. Health Commun 2007;21(01):97-99http://ezproxy.unal.edu.co/login? url=http://search.ebscohost.com/login.aspx?direct=true\&db=ed swss\&AN=000246767900010\&lang=es\&site=eds-live

3 Han GK, Zhang JM, Chu KR, Shen G. Self-other differences in H1N1 flu risk perception in a global context: a comparative study between the United States and China. Health Commun 2014;29 (02):109-123. Doi: 10.1080/10410236.2012.723267

4 World Health Organization. World Health Organization Outbreak Communication Planning Guide. 2008:1-19http://www.who.int/ ihr/elibrary/WHOOutbreakCommsPlanngGuide.pdf

5 Waisbord S. Cuando la salud es titular: dengue, gripe AH1N1 y ciclos “mediáticos-epidémicos.". Folios 2010;23:93-104

6 Obregon R, Arroyabe J, Barrios M. Cubrimiento periodístico de la gestión del riesgo en la Subregión Andina: discursos periodísticos y perspectivas desde la co.... Folios2010:23

7 Bonilla-Mahecha LP, Bonilla N, Pardo-Turriago R. Aportes para la comprensión e investigación del campo de la comunicación y salud: Experiencia desde el diáologo de saberes en la Universidad Nacional de Colombia. In: Congreso Internacional de La Asociación Española de Investigación En Comunicación. 2018 